\title{
De Novo modeling of Envelope 2 protein of HCV isolated from Pakistani patient and epitopes prediction for vaccine development
}

\author{
Samia Afzal, Muhammad Idrees ${ }^{*}$ and Mazhar Hussain
}

\begin{abstract}
Hepatitis C virus (HCV) is a universal health issue and a significant risk factor leading to hepatocellular carcinoma. HCV has infected approximately 170 million individuals worldwide. It is a member of Flaviviridae with positive sense RNA genome. In the absence of any effective vaccine against HCV, pegylated interferon with ribavirin is the standard of treatment against HCV infection. In this study, sequence and structural analysis of envelope 2 (E2) protein was performed which was isolated from patients of HCV genotype 3a in Pakistan. Then, epitopes were predicted which were specific for both B-cells and T-cells. Later, conservancy of epitopes was checked with the HCV $3 a$ and 1a sequences from different countries. A total of 6 conserved epitopes were found from extra-membranous regions of E2 protein. Presence of conserved epitopes in E2 protein generates the possibility that these epitopes can be used to elicit the immune response against HCV.
\end{abstract}

Keywords: HCV, E2 protein, Epitopes, Modeling

\section{Introduction}

Hepatitis $\mathrm{C}$ virus (HCV) is a universal health issue and a significant risk factor developing hepatocellular carcinoma (HCC). World Health Organization reported that hepatic cancer caused by HCV scored about 300,000 deaths in 2004 only [1]. It has affected approximately 170 million individuals worldwide [2]. In United States, $\mathrm{HCV}$ is most common blood-borne infection with over 4 million individuals infected [3]. According to a recent study, an alarming 17 million people in Pakistan are infected with HCV and about $8-10 \%$ of individuals are carriers of HCV pathogen [4]. Prevalence analysis of Pakistani population shows that HCV genotype $3 \mathrm{a}$ is the most common genotype in all provinces except in Balochistan where HCV genotype 1a is most prevalent [5].

$\mathrm{HCV}$ is a member of Flaviviridae and closely related to Dengue, West Nile and Yellow Fever virus. It has a positive sense single stranded RNA genome of about $9.6 \mathrm{~kb}$ size [6]. HCV genome encodes a large polyprotein of 3010 to 3033 amino acid residues [7,8]. This polyprotein

\footnotetext{
* Correspondence: idreeskhan@cemb.edu.pk

Division of Molecular Virology, National Centre of Excellence in Molecular Biology, University of the Punjab, 87-West Canal Bank Road Thokar Niaz Baig, Lahore 53700, Pakistan
}

is subsequently cleaved into four structural (Core, E1, E2 and P7) and 6 non structural proteins (NS2, NS3, NS4A, NS4B, NS5A and NS5B) [9].

However, the structural details of the HCV virus are still elusive [10], but it is known that infectious viral particles contains lipid envelope and glycoproteins E1 and E2, close to the surface [11]. E2 is highly glycosylated with most of the glycosylation sites well conserved [12]. In addition to these conserved residues, E2 has hypervariable regions which vary up to $80 \%$ among $\mathrm{HCV}$ of different genotypes and even between the subtypes of same genotype [13]. However, E2 protein interacts with DC-SIGN and L-SIGN (mannose binding proteins) but detailed mechanism of viral entry is unclear. It is suggested that glycosylated motifs of E2 protein interacts with surface receptor enabling the viral entry into the cell [14]. Hence, E2 protein is a potent target to stop viral entry into healthy cells [15].

Currently, the pegylated interferon (IFN) alpha, separately or in combination with ribavirin, is standard $\mathrm{HCV}$ treatment [16-18]. The efficacy of IFN treatment depends on many factors related to viral genotype and patient's health status. Clinical studies show that in 30-50\% of cases $\mathrm{HCV}$ remain non-responsive to IFN treatment 
and there might be a number of serious adverse events associated with treatment [9]. In spite of advancements in drug designing technologies, there is still no vaccine against HCV. Variability across the HCV genotypes is also a significant hindrance in vaccine development.

For the development of potential inhibitors against envelope proteins, it is required to have knowledge of sequence and structure of protein. With the development of computational biology, novel approaches have been developed to get insights from biological data. This study was designed to isolate E2 glycoprotein sequence from HCV genotype $3 \mathrm{a}$ infected patient; and to predict and to analyze the epitopes related to B-cells and Tcells. The conservation and variability analysis was included to find spectrum of activity of epitopes in $\mathrm{HCV}$ genotype $3 \mathrm{a}$ and $1 \mathrm{a}$.

\section{Methodology}

\section{Source of serum samples}

$\mathrm{HCV}$ genotype 3a samples were collected from patients diagnosed with $\mathrm{HCV}$ at Molecular Diagnostics Lab. CEMB, Lahore. The informed consent was taken from the patients and blood sample was taken according to provision of Ethical Committee, Molecular Virology Division, National Centre of Excellence in Molecular Biology, Lahore. The patients were selected on the basis of elevated serum ALT and AST levels at least for six months, histological examination, and detection of serum HCV RNA anti-HCV antibodies (3rd generation ELISA).

\section{RNA isolation from serum, cDNA synthesis and sequencing of HCV E2 gene}

Total RNA was isolated from patients' serum samples using Gentra RNA isolation kit (Puregene, Minneapolis, MN55441 USA) according to the kit protocol. Then, extracted RNA was reverse transcribed to cDNA using MMLV-RTase (Moloney murine leukemia virus reverse transcriptase). By using the E2 specific primers, E2 gene was amplified using cDNA of $\mathrm{HCV}$ genotype 3a. For this, PCR protocol involved 35 cycling steps at annealing temperature $54^{\circ} \mathrm{C}$. The amplified PCR product was resolved using 1.2\% TAE agarose gel and molecular weight was compared with $1 \mathrm{~kb}$ DNA ladder. Then, DNA was purified from gel using QIA quick gel extraction kit (Qiagen, USA) using the kit protocol. Purified PCR product was cloned in pCR2.1-TOPO (TA vectors) obtained from Invitrogen, USA. Successful cloning was confirmed by PCR using E2 specific primers and by digestion of construct using EcoR 1 at $37^{\circ} \mathrm{C}$ for $1 \mathrm{hr}$. Later, a sequencing reaction was performed using BigDye ${ }^{\mathrm{Ts}}$ Terminator v3.0 sequencing kit (Applied Biosystems, Germany). Both positive and negative strands were sequenced at automated sequencer (Applied Biosystems

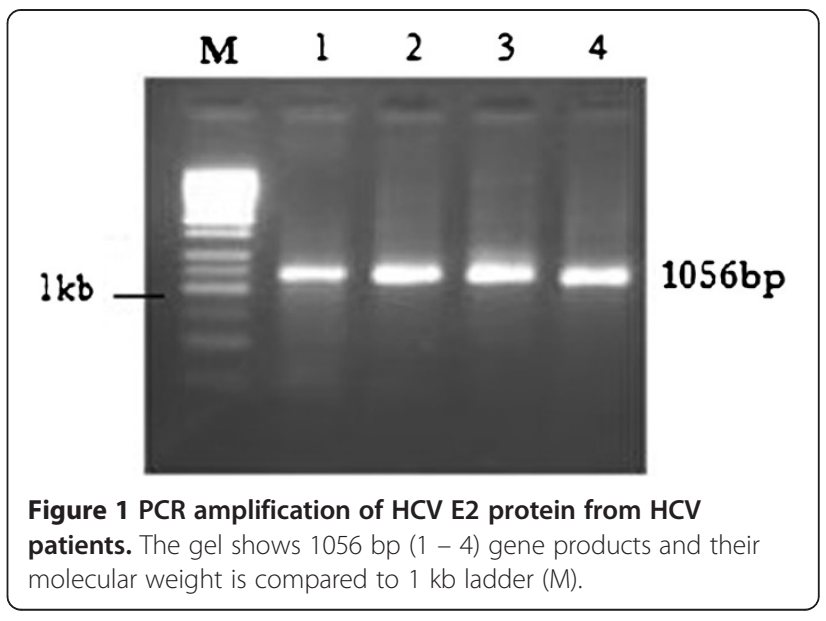

3700 DNA Analyzer, Germany). Then, the gene sequence was submitted at NCBI GenBank, having accession no. ADP55199.

\section{Sequence analysis of E2 protein}

HCV 3a E2 gene sequence ADP55199 was used for primary structure analysis and for the prediction of secondary as well as three-dimensional structure. The E2 gene sequence was in-silico translated to obtain primary structure (amino acid sequence) of protein. Primary structure parameters of E2 protein which include molecular weight, theoretical pI, atomic composition, extinction coefficient, estimated half-life, aliphatic index

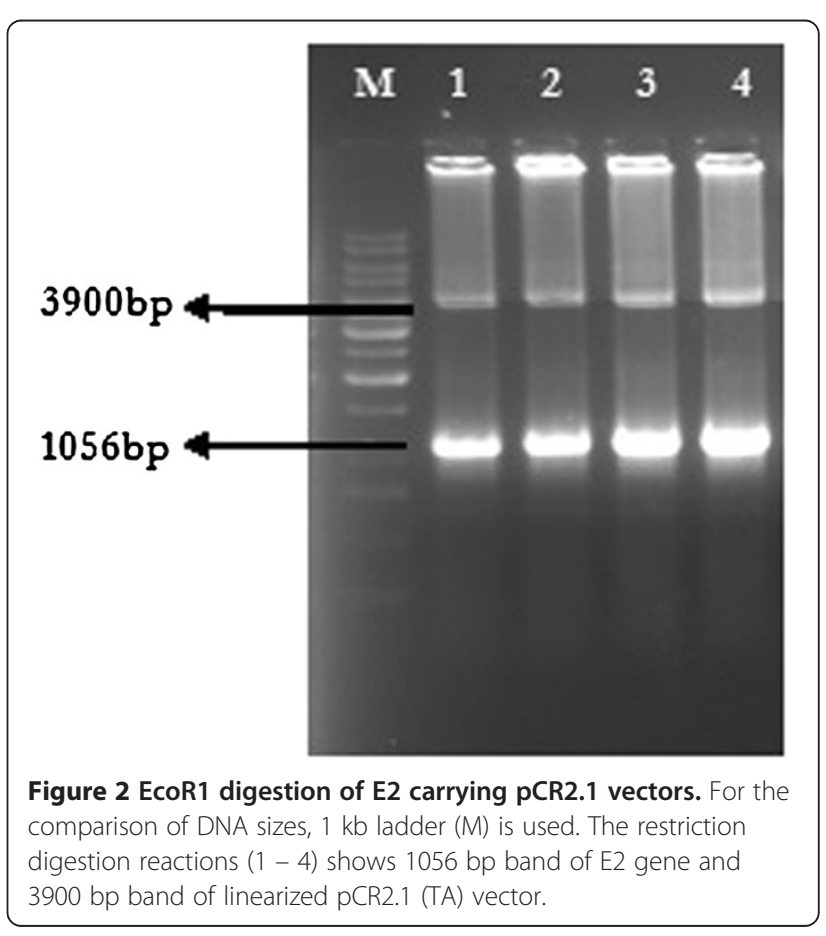




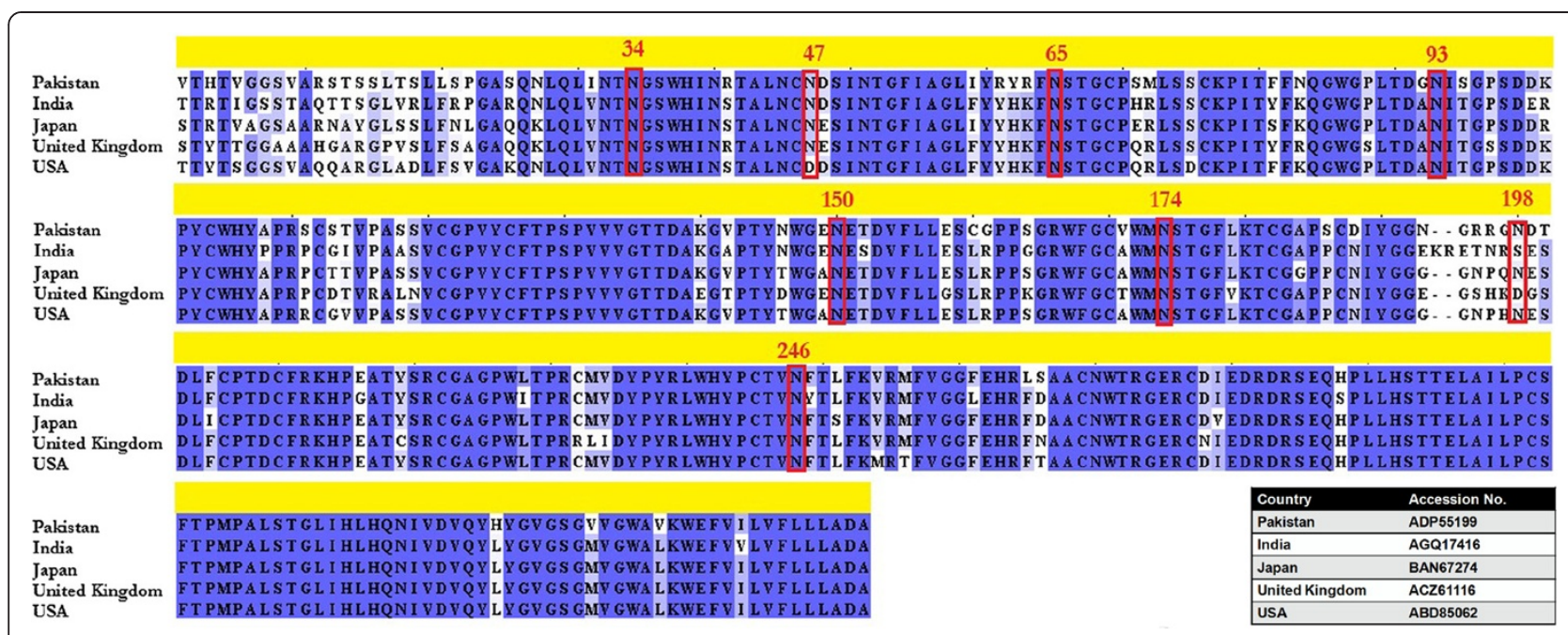

Figure 3 The glycosylation sites prediction and conservancy in these sites in E2 proteins from different regions of world.

and Grand average of hydropathicity (GRAVY) were computed using ProtParam online tool [19]. Secondary structure of the protein was analyzed using Jpred, Psipred and "Sequence Annotated by Structure" (SAS) tool [20-22]. Disulfide connectivity of the protein was checked using DiANNA tool which is a neural network application and predicts cysteine states of a protein [23]. The knowledge of cys-cys linkages is important in understanding the secondary and tertiary structure of protein because it plays significant role in fold stabilization. Glycosylation sites were predicted using NetNGlyc 1.0 server and their conservancy was checked using multiple sequence alignment by MEGA5.0 [24].

\section{De novo protein modeling and quality assessment}

For the prediction of three dimensional structure of E2 protein both homology modeling and de novo modeling approaches were used. For the homology modeling, BlastP was used for searching suitable template in Protein Data Bank (http://www.rcsb.org/pdb/home/home. do). In our search, the appropriate template was not found, so we used iTASSER server for de novo modeling of E2 protein [25]. Using iTASSER, five models were predicted and one best model was chosen for further structural analysis. The selection of model was done using three criteria: C-score, DFIRE2 energy profile [26] and stereochemical properties using PROCHECK tool [27]. The visual analysis of structure was done on SwissPDB-viewer [28] and Visual Molecular Dynamics (VMD) program [29].

\section{Epitopes prediction from E2 protein}

A systematic approach was employed for the prediction of potential epitopes in E2 protein. Vexijen 1.0 was used to determine overall antigenicity of E2 protein using cut- off value of 0.4 [30]. Then, topology of E2 protein was determined using TMHMM Server v 2.0 [31]. With the help of membrane topology data, E2 protein regions inside the membrane and transmembrane were eradicated from epitopes prediction. BCPRED server was used for the prediction of B-cell epitopes of the length of 12 amino acids [32]. For the prediction of T-cell epitopes ProPred was used with proteasome cleavage site filter of $5 \%$ threshold. In this analysis, 47 alleles of MHC-class I and 54 alleles of MHC-class II were included [33]. Once the B-cells and T-cells (MHC-class I and MHC-class II) epitopes were predicted, their antigenicity was checked using Vexijen. The antigenicity score of the predicted epitopes was checked using Vexijen v 1.0 server. Later, only antigenic epitopes were included in conservancy analysis.

\section{The conservancy of epitopes}

The E2 protein sequences of HCV genotype 3a and 1a were retrieved from NCBI sequence database. The $\mathrm{HCV} 3 \mathrm{a}$ sequences were from India (AGQ17416), Japan (BAN67274),

\section{Table 1 Predicted disulfide bonds}

\begin{tabular}{ll}
\hline Predicted bonds & \\
\hline $46-267$ & RTALNCNDSIN - RLSAACNWTRG \\
$69-104$ & FNSTGCPSMLS - DDKPYCWHYAP \\
$76-187$ & SMLSSCKPITF - CGAPSCDIYGG \\
$112-275$ & YAPRSCSTVPA - TRGERCDIEDR \\
$121-170$ & PASSVCGPVYC - GRWFGCWWMNS \\
$126-208$ & CGPVYCFTPSP - FCPTDCFRKHP \\
$160-204$ & FLLESCGPPSG - DTDLFCPTDCF \\
$220-300$ & ATYSRCGAGPW - LAILPCSFTPM \\
$230-243$ & WLTPRCMVDYP - LWHYPCTVNFT \\
\hline
\end{tabular}




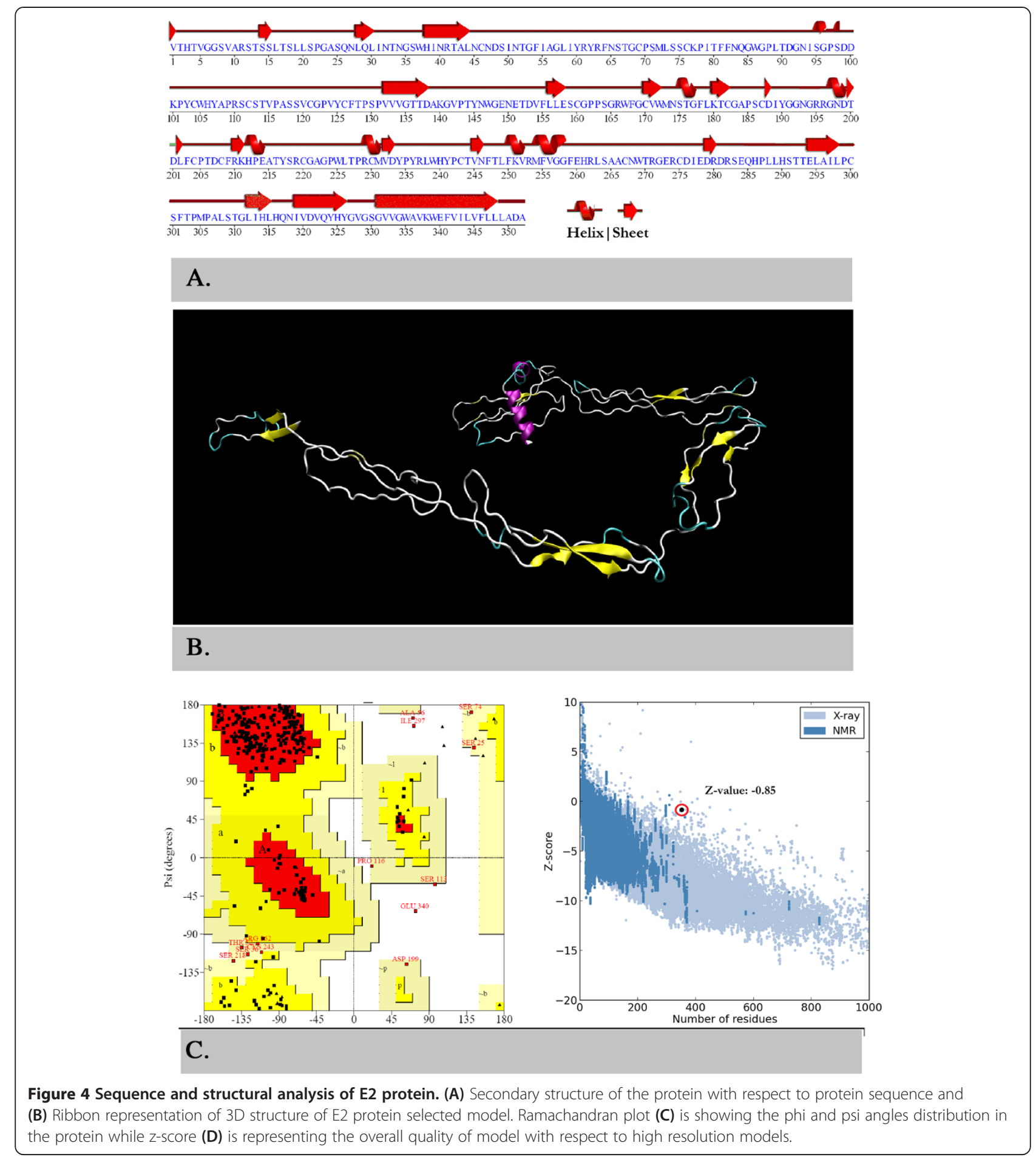

United Kingdom (ACZ61116) and USA (ABD85062) and HCV 1a sequences were from Pakistan (GU736411), USA (EU482831), United Kingdom (AY958057), France (AF529293) and Japan (AB520610). The conservancy and variability of the predicted antigenic epitopes was determined by "IEDB conservancy analysis tool" [34] in E2 protein sequences retrieved from different regions of world.
Then, all highly conserved epitopes were checked for their localization in predicted protein structure.

\section{Results and discussion}

CDNA synthesis and cloning of E2 protein

Serum samples collected from HCV genotype 3a positive individuals were processed for total RNA extraction. 
Complimentary DNA was prepared from RNA and then E2 gene was amplified using gene specific primers. The PCR product was checked on the gel and bands were observed at $1056 \mathrm{bp}$ position with reference to $1 \mathrm{~kb}$ DNA marker (Figure 1). DNA in the gel was used for ligation in pCR2.1 for TA cloning. TA cloning was verified by PCR using E2 gene specific primers and EcoR1 digestion. The restriction digestion with EcoR1 produced 2 bands for each sample (Figure 2). A band with $3.9 \mathrm{~kb}$ molecular weight indicates the linearized pCR2.1 and the other with $\sim 1.05 \mathrm{~kb}$ indicates the size of E2 gene. One sample was further processed for sequencing, structural and epitope prediction.

\section{Sequence analysis of E2 protein}

In this study, we have employed sequence analysis, de novo modeling and epitopes prediction from E2 protein isolated from Pakistani patient. Its estimated molecular weight is 38703.8 daltons and comprised of 352 amino acids. The $\mathrm{pI}$ and extinction coefficient of the protein were 6.86 and $80995 \mathrm{M}^{-1} \mathrm{~cm}^{-1}$, respectively. The protein has GRAVY score of -0.122 which indicates that protein is hydrophilic in nature. Glycosylation sites analysis revealed the presence of eight potential glycosylation sites in the protein (Figure 3). Among all, six sites at positions 34, 65, 93, 150, 174 and 246 were conserved in the sequences retrieved from various regions of the world. The protein also contains 9 disulfide bridges (Table 1) which renders extracellular stability in proteins $[35,36]$.

\section{De novo models and quality assessment}

Secondary structure of the protein shows that loops make the $78 \%$ of the protein while sheets and helices contribute $14 \%$ and $8 \%$ of the structure, respectively (Figure 4A). Using iTASSER server, five protein models were developed. The best model was selected by combined evaluation based on C-score (confidence score from iTASSER server), DFIRE2 energy profile and stereochemical properties using Ramachandran plot. It is very evident from Table 2 that Model 1 is best

Table 2 Assessment of iTASSER predicted E2 protein models

\begin{tabular}{llllll}
\hline & Model 1 & Model 2 & Model 3 & Model 4 & Model 5 \\
\hline I-TASSER (C-score) & -1.17 & -3.11 & -3.12 & -3.17 & -3.35 \\
DFIRE2 & -420.54 & -586.18 & -573.31 & -579.41 & -599.08 \\
PROCHECK & & & & & \\
Allowed & $82.1 \%$ & $69.4 \%$ & $72.9 \%$ & $69.8 \%$ & $70.4 \%$ \\
$\begin{array}{l}\text { Additionally } \\
\text { allowed }\end{array}$ & $13.7 \%$ & $19.6 \%$ & $17.9 \%$ & $19.6 \%$ & $20.3 \%$ \\
$\begin{array}{l}\text { Generously } \\
\text { allowed }\end{array}$ & $2.7 \%$ & $4.1 \%$ & $5.2 \%$ & $5.5 \%$ & $3.8 \%$ \\
\begin{tabular}{l} 
Disallowed \\
\hline
\end{tabular} & $1.4 \%$ & $6.9 \%$ & $4.1 \%$ & $5.2 \%$ & $5.5 \%$ \\
\hline
\end{tabular}

Table 3 B-cell epitopes with their antigenicity score

\begin{tabular}{llll}
\hline $\begin{array}{l}\text { Starting } \\
\text { position }\end{array}$ & Epitope & $\begin{array}{l}\text { BCPreds } \\
\text { score }\end{array}$ & $\begin{array}{l}\text { Antigenicity } \\
\text { score }\end{array}$ \\
\hline 160 & CGPPSGRWFGCV & $\mathbf{0 . 9 9 8}$ & $-\mathbf{0 . 7 5 6 9}$ \\
82 & FNQGWGPLTDGN & 0.969 & 0.9925 \\
188 & DIYGGNGRRGND & 0.965 & 1.4612 \\
136 & TTDAKGVPTYNW & 0.93 & 0.4148 \\
29 & QLINTNGSWHIN & 0.916 & 0.7087 \\
278 & EDRDRSEQHPLL & 0.892 & 0.5071 \\
108 & APRSCSTVPASS & $\mathbf{0 . 8 4 9}$ & $\mathbf{- 0 . 0 1 6 4}$ \\
221 & GAGPWLTPRCMV & 0.827 & 0.4537 \\
203 & FCPTDCFRKHPE & $\mathbf{0 . 8 1 9}$ & $\mathbf{- 0 . 1 9 6 3}$ \\
123 & PVYCFTPSPWW & 0.812 & 0.9481 \\
60 & YRYRFNSTGCPS & 0.806 & 0.9192 \\
43 & ALNCNDSINTGF & 0.679 & 0.8184 \\
95 & SGPSDDKPYCWH & 0.645 & 1.2504 \\
174 & NSTGGFLKTCGAP & $\mathbf{0 . 5 8 7}$ & $\mathbf{- 0 . 3 6 9 8}$ \\
301 & SFTPMPALSTGL & 0.56 & 0.6394 \\
234 & YPYRLWHYPCTV & 0.383 & 0.4361 \\
265 & AACNWTRGERCD & 0.311 & 0.4919 \\
252 & VRMFVGGFEHRL & $\mathbf{0 . 2 9 1}$ & $\mathbf{0 . 1 9 3 4}$ \\
\hline
\end{tabular}

Non-Antigens are shown in bold face.

among all others based on C-score and Ramachandran plot. In addition to this, secondary structures in threedimensional structure of Model1 have stronger correlation with predicted secondary structures with other methods (Figure 4B). As far as, Model1 stereochemical quality is concerned, it showed $82.1 \%$ amino acid residues in allowed and only $1.4 \%$ residues in disallowed region of Ramachandran plot (Figure 4C). However, its DFIRE2 score was not better than other 4 models but its $\mathrm{z}$-score $(-0.85)$ based on its overall energy was comparable with non-redundant set of high quality models (Figure 4D). On

Table 4 MHC Class I epitopes with their antigenicity scores

\begin{tabular}{|c|c|c|c|}
\hline $\begin{array}{l}\text { Starting } \\
\text { position }\end{array}$ & Peptide & Allele & $\begin{array}{l}\text { Antigenicity } \\
\text { score }\end{array}$ \\
\hline 81 & FFNQGWGPL & $\begin{array}{l}\text { HLA-A24, HLA-B_3801, HLA- } \\
\text { B_3902, HLA-B_5301, HLA- } \\
\text { B_5401, HLA-B_51, HLA-B_0702, } \\
\text { HLA-CW_0401, MHC-Kd }\end{array}$ & 1.0281 \\
\hline 128 & TPSPWVGT & $\begin{array}{l}\text { HLA-B_3501, HLA-B_5101, HLA- } \\
\text { B_5102, HLA-B_5103, MHC-Ld }\end{array}$ & 1.5496 \\
\hline 12 & STSSLTSLL & $\begin{array}{l}\text { HLA-B_3701, HLA-B_3902, } \\
\text { HLA-B_5801, HLA-B60, HLA- } \\
\text { B7, HLA-Cw_0602, MHC-Kb }\end{array}$ & 0.0685 \\
\hline 1 & VTHTVGGSV & $\begin{array}{l}\text { HLA-B_5103, HLA-B_5301, HLA- } \\
\text { B_51, HLA-B_5801, HLA-B61 }\end{array}$ & 0.2406 \\
\hline
\end{tabular}

Non-Antigens are shown in bold face. 
Table 5 MHC Class II epitopes with antigenicity scores

\begin{tabular}{|c|c|c|c|}
\hline $\begin{array}{l}\text { Starting } \\
\text { position }\end{array}$ & Peptide & Allele & $\begin{array}{l}\text { Antigenicity } \\
\text { score }\end{array}$ \\
\hline 28 & LQLINTNGS & $\begin{array}{l}\text { DRB1_0101, DRB1_0102, DRB1_0301, DRB1_0305, DRB1_0306, DRB1_0307, DRB1_0308, } \\
\text { DRB1_0309, DRB1_0311, DRB1_0401, DRB1_0402, DRB1_0404, DRB1_0405, DRB1_0408, } \\
\text { DRB1_0410, DRB1_0421, DRB1_0423, DRB1_0426, DRB1_0802, DRB1_0804, DRB1_0806, } \\
\text { DRB1_0813, DRB1_1101, DRB1_1102, DRB1_1104, DRB1_1106, DRB1_1107, DRB1_1114, } \\
\text { DRB1_1120, DRB1_1121, DRB1_1128, DRB1_1301, DRB1_1302, DRB1_1304, DRB1_1305, } \\
\text { DRB1_1307, DRB1_1311, DRB1_1321, DRB1_1322, DRB1_1323, DRB1_1327, DRB1_1328, } \\
\text { DRB1_1506, DRB5_0101, DRB5_0105 }\end{array}$ & -0.2636 \\
\hline 64 & FNSTGCPSM & DRB1_0101, DRB1_0701, DRB1_0703, & 0.6645 \\
\hline 179 & LKTCGAPSC & DRB1_0101, DRB1_0102, & -0.2346 \\
\hline 31 & INTNGSWHI & DRB1_0102, DRB1_0402, DRB1_0701, DRB1_0703, & 1.0793 \\
\hline 124 & VYCFTPSPV & DRB1_0102, DRB1_0423, DRB1_1501, DRB1_1506 & 1.4124 \\
\hline 154 & VFLLESCGP & DRB1_0102, DRB1_0404, DRB1_0408, DRB1_0410, DRB1_0421, DRB1_0423, & -1.3987 \\
\hline 58 & LIYRYRFNS & $\begin{array}{l}\text { DRB1_0301, DRB1_0305, DRB1_0309, DRB1_0801, DRB1_0802, DRB1_0804, DRB1_0806, } \\
\text { DRB1_0813, DRB1_0817, DRB1_1101, DRB1_1102, DRB1_1104, DRB1_1106, DRB1_1107, } \\
\text { DRB1_1114, DRB1_1120, DRB1_1121, DRB1_1301, DRB1_1302, DRB1_1304, DRB1_1307, } \\
\text { DRB1_1311, DRB1_1322, DRB1_1323, DRB1_1327, DRB1_1328, DRB1_1501, DRB1_1506 }\end{array}$ & -0.051 \\
\hline 72 & MLSSCKPIT & DRB1_0301, DRB1_0305, DRB1_0309, DRB1_1107 & -0.3409 \\
\hline 132 & WVGTTDAK & DRB1_0301, DRB1_0305, DRB1_0306, DRB1_0307, DRB1_0308, DRB1_0311, DRB1_1107, & 1.8645 \\
\hline 37 & WHINRTALN & $\begin{array}{l}\text { DRB1_0305, DRB1_0309, DRB1_0401, DRB1_0405, DRB1_0408, DRB1_0410, DRB1_0421, } \\
\text { DRB1_0426, DRB1_0801, DRB1_0802, DRB1_0806, DRB1_0817, DRB1_1101, DRB1_1114, } \\
\text { DRB1_1120, DRB1_1128, DRB1_1302, DRB1_1304, DRB1_1305, DRB1_1307, DRB1_1321, } \\
\text { DRB1_1323 }\end{array}$ & 0.3868 \\
\hline 62 & YRFNSTGCP & $\begin{array}{l}\text { DRB1_0305, DRB1_0309, DRB1_0401, DRB1_0405, DRB1_0408, DRB1_0421, DRB1_0426, DRB1_0801, } \\
\text { DRB1_0802, DRB1_1101, DRB1_1120, DRB1_1128, DRB1_1302, DRB1_1305, DRB1_1307, DRB1_1321, }\end{array}$ & 0.9493 \\
\hline 105 & WHYAPRSCS & $\begin{array}{l}\text { DRB1_0305, DRB1_0306, DRB1_0307, DRB1_0308, DRB1_0309, DRB1_0311, DRB1_0801, DRB1_0802, } \\
\text { DRB1_0813, DRB1_1101, DRB1_1107, DRB1_1114, DRB1_1120, DRB1_1128, DRB1_1302, DRB1_1305, } \\
\text { DRB1_1307, DRB1_1323 }\end{array}$ & 0.5807 \\
\hline 172 & WMNSTGFLK & DRB1_0305, DRB5_0101, DRB5_0105 & 0.1523 \\
\hline 169 & WTRGERCDI & DRB1_0305, DRB1_0309, & 0.9138 \\
\hline 54 & FIAGLIYRY & DRB1_0309, & 0.4518 \\
\hline 127 & FTPSPWW & DRB1_0309, DRB1_0421 & 1.3553 \\
\hline 239 & WHYPCTVNF & DRB1_0309, DRB1_0405, DRB1_0421, DRB1_0703 & 0.7657 \\
\hline 167 & WFGCVWMNS & DRB1_0401, DRB1_0408, DRB1_1101 & 0.2989 \\
\hline 60 & YRYRFNSTG & $\begin{array}{l}\text { DRB1_0402, DRB1_0405, DRB1_0408, DRB1_0421, DRB1_0801, DRB1_0802, DRB1_0806, DRB1_0813, } \\
\text { DRB1_0817, DRB1_1120, DRB1_1302, DRB1_1502 }\end{array}$ & 1.5053 \\
\hline 247 & FTLFKVRMF & $\begin{array}{l}\text { DRB1_0402, DRB1_0701, DRB1_0703, DRB1_0801, DRB1_0802, DRB1_1101, DRB1_1102, DRB1_1114, } \\
\text { DRB1_1120, DRB1_1121, DRB1_1128, DRB1_1301, DRB1_1302, DRB1_1304, DRB1_1305, DRB1_1307, } \\
\text { DRB1_1323, DRB1_1327, DRB1_1328 }\end{array}$ & 0.0943 \\
\hline 256 & VGGFEHRLS & $\begin{array}{l}\text { DRB1_0402, DRB1_1102, DRB1_1114, DRB1_1120, DRB1_1121, DRB1_1301, DRB1_1302, DRB1_1304, } \\
\text { DRB1_1322, DRB1_1323, DRB1_1327, DRB1_1328 }\end{array}$ & 1.3166 \\
\hline 252 & VRMFVGGFE & $\begin{array}{l}\text { DRB1_0405, DRB1_0410, DRB1_0801, DRB1_0806, DRB1_0817, DRB1_1304, DRB1_1321, } \\
\text { DRB1_1501, DRB1_1502, DRB1_1506 }\end{array}$ & -0.4355 \\
\hline 147 & WGENETDVF & DRB1_0421 & -0.0038 \\
\hline 125 & YCFTPSPW & DRB1_0701, DRB1_0703 & 1.2112 \\
\hline 241 & YPCTVNFTL & DRB1_0701, DRB1_0703 & 0.9270 \\
\hline 259 & FEHRLSAAC & DRB1_0801, DRB1_0802, DRB1_0813 & 1.8179 \\
\hline 277 & IEDRDRSEQ & DRB1_0813 & 0.5283 \\
\hline 103 & YCWHYAPRS & DRB1_1114, DRB1_1120, DRB1_1302, DRB1_1323 & 1.3992 \\
\hline 190 & YGGNGRRGN & DRB1_1114, DRB1_1120, DRB1_1302, DRB1_1304, DRB1_1323 & 2.2420 \\
\hline 209 & FRKHPEATY & DRB1_1114, DRB1_1120, DRB1_1302, DRB1_1323, & 0.8056 \\
\hline 236 & YRLWHYPCT & DRB1_1502, DRB1_1506 & 0.5131 \\
\hline
\end{tabular}


Table 5 MHC Class II epitopes with antigenicity scores (Continued)

\begin{tabular}{llll}
\hline 245 & VNFTLFKVR & DRB5_0101, DRB5_0105 & 0.9765 \\
59 & IYRYRFNST & DRB1_1501, DRB1_1502, DRB1_1506 & 0.9405 \\
55 & IAGLIYRYR & DRB5_0101, DRB5_0105 & 1.4380 \\
\hline
\end{tabular}

Non-Antigens are shown in bold face.

these bases, model 1 was chosen for the structural analysis of E2 protein.

\section{Epitopes prediction for the vaccine development}

Humoral and cellular immunity are the two arms of immunity provided by B-cells and T-cells, respectively. The recognition of pathogenic epitopes by B-cell and T-cells lies at the heart of immune response. Such recognition starts the mechanism of activation of humoral and cellular response and leads to ultimate destruction of pathogenic organism [35]. Epitopes are principal components of both subunit and poly-epitopic vaccines. Thus, it is a pivotal challenge for immune-informatics to accurately predict the B-cell and T-cells epitopes [37].

It is important for a protein to expose in outside environment to interact with soluble antibodies, B-cells and T-cells. So, the membrane topology of the E2 protein was determined using TMHMM server. In this analysis, a total of 2 transmembrane helices with, 23 amino acids each, were predicted. First helix spans from residue 293 to residue 315 while second helix spans from residue 328 to residue 350 . A large 1 to 292 portion of the protein was outside of the membrane and a small loop like structure was present inside the membrane. For the prediction of B-cell and T-cell epitopes, only extramembranous region was selected. The antigenicity score of selected $(1-292)$ region was 0.4911 which indicated that this region as a probable antigen.

\section{B-cell epitopes prediction}

B-cell epitopes are important to elicit humoral immune response. B-Cell epitopes were predicted using BCPred server. Using the configuration of 12 amino acids, 18 epitopes were predicted using extra-membranous region of the protein (Table 3). Then, all epitopes were checked for antigenicity using Vexijen server. Among them 13 were having antigenicity score greater than 0.4 threshold value for antigens while 5 were below this limit. Epitope DIYGGNGRRGND present at position 188 had highest antigenicity score. A higher antigenicity score indicates the better binding affinity between receptor and epitope [36].

\section{$T$ cell epitopes prediction}

T-cell epitopes are presented on either MHC class I or MHC class II which play significant role in cell-mediated immunity $[38,39]$. For the prediction of T-cell epitopes, we used ProPred server with 47 alleles for MHC class I and 54 alleles for MHC class II. A total of 4 epitopes were predicted for MHC class I in extra-membranous region while only 2 epitopes, FFNQGWGPL and TPSPVVVGT, showed antigenicity score greater than antigenicity threshold (0.4) (Table 4). Similarly, 34 epitopes were predicted for MHC class II and 24 among them were found to be antigenic (Table 5). Out of 24 antigenic peptides, eleven showed antigenicity score $>1.000$ which is considered good in terms of stability of receptor and epitope interaction.

\section{Conservancy and structural analysis of predicted epitopes}

The assessment of epitope conservation is important in vaccine design because a higher level of conservation ensure broader protection against multiple strains of a pathogen [34]. The conservancy of the predicted epitopes was determined using E2 proteins from $\mathrm{HCV} 3 \mathrm{a}$ and HCV 1a from various regions of the world. For this, 3a sequence from India, Japan, United Kingdom and USA; and 1a sequence from Pakistan, France, Japan, UK and USA were downloaded from NCBI database. The IEDB conservancy analysis was used to determine conserved epitopes among all selected E2 sequences from genotypes 3a and 1a (Additional file 1: Table S1). Separate analysis of $\mathrm{HCV}$ genotype $3 \mathrm{a}$ and 1a epitopes showed conservancy in 9 epitope sequences each. Further analysis showed that six epitopes were highly conserved for both HCV genotype 3a and 1a (Table 6). Among 6 conserved epitopes, a B-cell epitope and an MHC-class I (T-cell) epitope was conserved at positions 123 and 128, respectively. Furthermore, four MHC-class II (T-cell) epitopes were found conserved at positions 124, 125, 127 and 236. This indicates that E2 protein can elicit not only humoral immune response but also helper-T cells and cytotoxic-T cells too. The structural analysis of E2

Table 6 Highly conserved epitopes from E2 of 3a and 1a

\begin{tabular}{ll}
\hline Epitopes & B-cell/T-cell \\
\hline PVYCFTPSPWV & B-cell \\
TPSPWVGT & T-cell (HLA-B_3501, HLA-B_5101, HLA-B_5102, \\
& HLA-B_5103, MHC-Ld) \\
VYCFTPSPV & T-cell (DRB1_0102, DRB1_0423, DRB1_1501, DRB1_1506) \\
FTPSPWWG & T-cell (DRB1_0309, DRB1_0421) \\
YCFTPSPW & T-cell (DRB1_0701, DRB1_0703) \\
YRLWHYPCT & T-cell (DRB1_1502, DRB1_1506) \\
\hline
\end{tabular}




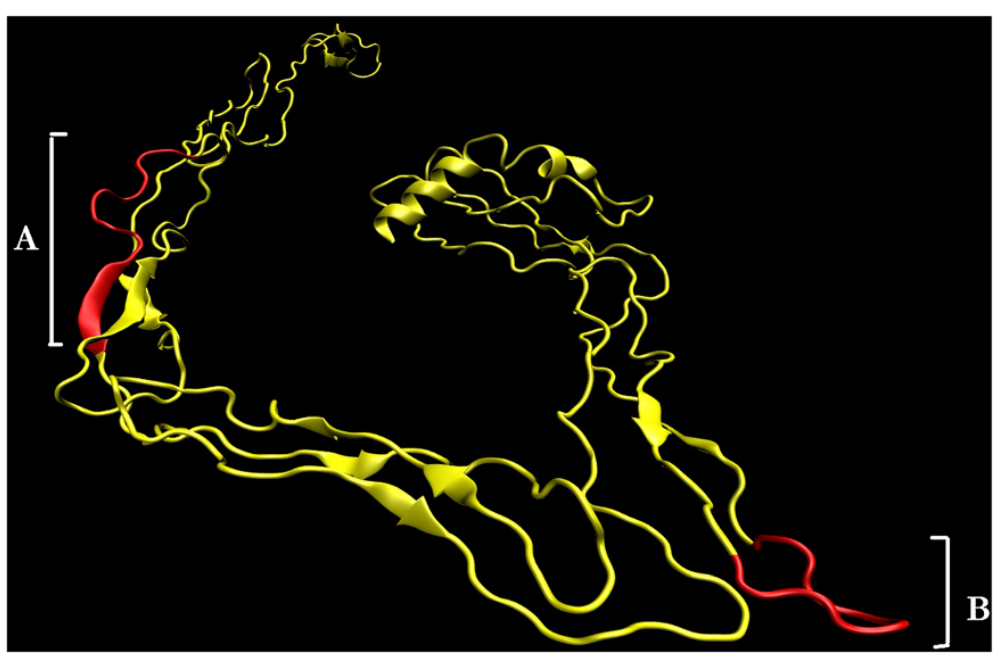

Figure 5 Structural analysis of highly conserved epitopes in HCV E2 protein. The structure revealed that it contains overlapping epitopes (A) region from 123 - 136 related to B-cells and T-cells. On the other hand, a single epitope is also present at position 236 (B) which is related to T-cell.

protein showed that all epitopes were exposed to the surface (Figure 5). Surface exposure of epitopes is important for interaction with respective immune cell receptors $[35,36,40]$.

Moreover, it is generally desirable that a vaccine formulation may have one or more B-cell and T-cell epitopes because a wide immune response can efficiently eradicate the invading pathogen. Sometimes, a small protein motif with overlapping epitopes for B-cells and T-cells can stimulate the humoral and cell-mediated immune response. The conserved epitopes in HCV E2 protein showed that the loop-sheet motif from 123 to 136 region contains 5 overlapping epitopes for both B-cells and T-cells (Figure 5). This motif has one B-cell and 4. T-cell epitopes. T-cell epitopes include one MHC class I specific and three MHC class II specific epitopes. Hence, this motif of 13 amino acids can induce broad immune response against $\mathrm{HCV}$ pathogen.

\section{Conclusion}

$\mathrm{HCV}$ is prevalent worldwide and there is no vaccine developed against this virus. There are multiple antigenic components which can be used for vaccine development. In Pakistan, HCV genotype 3a is most common followed by 1a. The sequence, structural and epitope analysis has revealed a number of conserved epitopes in both $3 \mathrm{a}$ and 1a genotypes. These epitopes may not only help in diagnosing the pathogens but also may help in developing vaccine against HCV $3 \mathrm{a}$ and 1a. Presence of overlapping epitopes generates the hope that a small fragment of peptide in vaccine formulation can elicit broad immune response and may result in efficient clearing of pathogen.

\section{Additional file}

Additional file 1: Table S1. Conservancy of E2 protein epitopes with HCV $3 a$ and HCV la sequences from various countries.

Competing interests

The authors declare that they have no competing interests.

\section{Authors' contributions}

MI conceived of the study. SA performed the experimental work, organised the data and drafted the manuscript. $\mathrm{MH}$ performed the evolutionary and statistical analysis. All the authors read and approved the final manuscript.

Received: 17 January 2014 Accepted: 26 March 2014 Published: 7 May 2014

\section{References}

1. Jafri W, Subhan A: Hepatitis C in Pakistan: magnitude, genotype, disease characteristics and therapeutic response. Tropical Gastroenterol 2010, 29:194-201.

2. Al Naamani K, Al Sinani S, Deschenes M: Epidemiology and treatment of hepatitis C genotypes 5 and 6. Can J Gastroenterol 2013, 27:8-12.

3. Alter MJ: Epidemiology of hepatitis C virus infection. W J Gastroenterol $2007,13: 2436$

4. Hussain $\mathrm{A}$, Idrees M: The first complete genome sequence of HCV-1a from Pakistan and a phylogenetic analysis with complete genomes from the rest of the world. Virol I 2013, 10:211.

5. Ashfaq UA, Javed T, Rehman S, Nawaz Z, Riazuddin S: An overview of HCV molecular biology, replication and immune responses. Virol J 2011, 8:161.

6. Jhaveri R, Kundu P, Shapiro AM, Venkatesan A, Dasgupta A: Effect of heptitis $C$ virus core protein on cellular gene expression: specific inhibition of cyclooxygenase 2. J Infec Dis 2005, 191:1498-1506.

7. Brass V, Moradpour D, Blum HE: Molecular virology of hepatitis $C$ virus (HCV): 2006 update. Int J Med Sci 2006, 3:29.

8. Penin F, Dubuisson J, Rey FA, Moradpour D, Pawlotsky JM: Structural biology of hepatitis C virus. Hepatol 2004, 39:5-19.

9. Afzal S, Idrees M, Ali M, llyas M, Hussain A, Akram M, Butt S, Saleem S, Rehman I, Ali L, Shahid M: Envelope 2 protein phosphorylation sites S75 \& 277 of hepatitis $C$ virus genotype $1 \mathrm{a}$ and interferon resistance: A sequence alignment approach. Virol J 2011, 8:71. 
10. Budkowska A: Mechanism of cell infection with hepatitis C virus (HCV)-a new paradigm in virus-cell interaction. Pol J Microbiol 2009, 58:93-98.

11. Idrees S, Ashfaq UA, Idrees N: Development of global consensus sequence of HCV glycoproteins involved in viral entry. Theor Biol Med Model 2013, 10:24.

12. Bartosch B, Dubuisson J, Cosset F-L: Infectious hepatitis C virus pseudoparticles containing functional E1-E2 envelope protein complexes. J Exp Med 2003, 197:633-642.

13. Ashfaq UA, Masoud MS, Nawaz Z, Riazuddin S: Glycyrrhizin as antiviral agent against Hepatitis C Virus. J Trans Med 2011, 9:112.

14. Gardner JP, Durso RJ, Arrigale RR, Donovan GP, Maddon PJ, Dragic T, Olson WC: L-SIGN (CD 209 L) is a liver-specific capture receptor for hepatitis C virus. Proceed Nat Acad Sci 2003, 100:4498-4503.

15. Helle F, Dubuisson J: Hepatitis C virus entry into host cells. Cell and Mol Life Sci 2008, 65:100-112.

16. Aman W, Mousa S, Shiha G, Mousa SA: Current status and future directions in the management of chronic hepatitis C. Virol J 2012, 9:57.

17. Susser S, Welsch C, Wang Y, Zettler M, Domingues FS, Karey U, Huges E, Ralston R, Tong X, Herrman E, Zeuzem S, Sarrazin C: Characterization of resistance to the protease inhibitor boceprevir in hepatitis $C$ virusinfected patients. Hepato/ 2009, 50:1709-1718.

18. Shiffman ML, Esteban R: Triple therapy for HCV genotype 1 infection: telaprevir or boceprevir? Liver Int 2012, 32:54-60.

19. Gasteiger E, Hoogland C, Gattiker A, Wilkins MR, Appel RD, Bairoch A: Protein Identification and Analysis Tools on the ExPASy Server. In The Proteomics Protocols Handbook. Totowa: Springer; 2005:571-607.

20. Cuff JA, Clamp ME, Siddiqui AS, Finlay M, Barton GJ: JPred: a consensus secondary structure prediction server. Bioinform 1998, 14:892-893.

21. McGuffin LJ, Bryson K, Jones DT: The PSIPRED protein structure prediction server. Bioinforma 2000, 16:404-405.

22. Milburn D, Laskowski RA, Thornton JM: Sequences annotated by structure: a tool to facilitate the use of structural information in sequence analysis. Prot Eng 1998, 11:855-859.

23. Ferrè $F$, Clote $P$ : DiANNA: a web server for disulfide connectivity prediction. Nucleic Acids Res 2005, 33:W230-W232.

24. Tamura K, Peterson D, Peterson N, Stecher G, Nei M, Kumar S: MEGA5: molecular evolutionary genetics analysis using maximum likelihood, evolutionary distance, and maximum parsimony methods. Mol Biol Evol 2011, 28:2731-2739.

25. Zhang Y: I-TASSER server for protein 3D structure prediction. BMC Bioinform 2008, 9:40.

26. Yang $Y$, Zhou $Y$ : Specific interactions for $a b$ initio folding of protein terminal regions with secondary structures. Proteins 2008, 72:793-803.

27. Laskowski RA, MacArthur MW, Moss DS, Thornton JM: PROCHECK: a program to check the stereochemical quality of protein structures. J Appl Cryst 1993, 26:283-291.

28. Guex N, Peitsch MC: SWISS-MODEL and the Swiss-Pdb Viewer: an environment for comparative protein modeling. Electrophor 1997, 18:2714-2723.

29. Humphrey W, Dalke A, Schulten K: VMD: visual molecular dynamics. J Mol Graph 1996, 14:33-38.

30. Doytchinova IA, Flower DR: VaxiJen: a server for prediction of protective antigens, tumour antigens and subunit vaccines. BMC Bioinform 2007, 8:4.

31. Krogh A, Larsson B, Von Heijne G, Sonnhammer EL: Predicting transmembrane protein topology with a hidden Markov model: application to complete genomes. J Mol Biol 2001, 305:567-580.

32. EL-Manzalawy $Y$, Dobbs D, Honavar V: Predicting linear B-cell epitopes using string kernels. J Mol Recog 2008, 21:243-255.

33. Singh H, Raghava G: ProPred: prediction of HLA-DR binding sites. Bioinform 2001, 17:1236-1237.

34. Bui H-H, Sidney J, Li W, Fusseder N, Sette A: Development of an epitope conservancy analysis tool to facilitate the design of epitope-based diagnostics and vaccines. BMC Bioinform 2007, 8:361.

35. Lazoura E, Lodding J, Farrugia W, Ramsland PA, Stevens J, Wilson IA, Pietersz GA, Apostolopoulos V: Enhanced major histocompatibility complex class I binding and immune responses through anchor modification of the non-canonical tumour-associated mucin 1-8 peptide. Immunol 2006, 119:306-316.

36. Niemann HH, Schubert W-D, Heinz DW: Adhesins and invasins of pathogenic bacteria: a structural view. Microb Infect 2004, 6:101-112.

37. Pietersz GA, Pouniotis DS, Apostolopoulos V: Design of peptide-based vaccines for cancer. Curr Med Chem 2006, 13:1591-1607.
38. Bhasin M, Raghava G: A hybrid approach for predicting promiscuous MHC class I restricted T cell epitopes. J Biosci 2007, 32:31-42.

39. Sturniolo T, Bono E, Ding J, Raddrizzani L, Tuereci O, Sahin U, Braxenthaler M, Gallazzi F, Protti MP, Sinigaglia F, Hammer J: Generation of tissuespecific and promiscuous HLA ligand databases using DNA microarrays and virtual HLA class II matrices. Nat Biotechnol 1999, 17:555-561.

40. Remaut $\mathrm{H}$, Waksman $\mathrm{G}$ : Structural biology of bacterial pathogenesis. Curr Opin Struct Biol 2004, 14:161-170.

doi:10.1186/1479-5876-12-115

Cite this article as: Afzal et al:: De Novo modeling of Envelope 2 protein of HCV isolated from Pakistani patient and epitopes prediction for vaccine development. Journal of Translational Medicine 2014 12:115.

\section{Submit your next manuscript to BioMed Central and take full advantage of:}

- Convenient online submission

- Thorough peer review

- No space constraints or color figure charges

- Immediate publication on acceptance

- Inclusion in PubMed, CAS, Scopus and Google Scholar

- Research which is freely available for redistribution

Submit your manuscript at www.biomedcentral.com/submit
C) Biomed Central 